\title{
Synthesis of Thianthrene Derivatives Linked by Carbon Chains
}

Md. Chanmiya Sheikh,* Takasi Iwasawa, Akitaka Nakajima, Atsutaka Kitao, Noritatsu Tsubaki, Ryuta Miyatake, Toshiaki Yoshimura, ${ }^{*}$ Hiroyuki Morita*

Department of Applied Chemistry, Faculty of Engineering, University of Toyama, 3190 Gofuku, Toyama 930-8555, Japan Fax+81(76)4456850; E-mail: chansheikh@yahoo.com; E-mail: yosimura@eng.u-toyama.ac.jp; E-mail: moritah@pb.ctt.ne.jp Received: 16.07.2013; Accepted after revision: 02.10.2013

\begin{abstract}
Dithianthren-1-ylmethanol and 1,1'-methylenedithianthrene were prepared and their reactions were studied. Lithiation of $1,1^{\prime}$-methylenedithianthrene took place on the methylene carbon rather than on the thianthrene framework, and when the lithiated derivative was allowed to react with thianthren-1-ylcarbaldehyde, sterically hindered 1,2,2-trithianthren-1-ylethanol was obtained in good yield. The structures of 1,1'-methylenedithianthrene and 1,2,2-trithianthren-1-ylethanol were confirmed by X-ray crystallography. To clarify the nature and reactivity of thianthrene derivatives, we also prepared 1,6-(thianthren-1,9-diyl)hexane-1,6-diol $(5,6,7,8,9,10$-hexahydro-1,14-epithiodibenzo[ $b, j]$ thiacycloundecine-5,10-diol) as a model compound in which the 1- and 9-positions of thianthrene are bridged by a carbon chain.
\end{abstract}

Key words: heterocycles, polycycles, macrocycles, sulfur

Thianthrene is known to fold along its $\mathrm{S}-\mathrm{S}$ axis ${ }^{1}$ (boat form) in a similar manner to 1,4 -dithiane, ${ }^{2}$ and to exist as butterfly structure in its equilibrium state of two flip-flop conformational isomers ${ }^{3}$ with an inherently low energy barrier. The sulfur chemistry of thianthrene, such as its sulfoxide, sulfone, and sulfilimine derivatives, has been studied widely. ${ }^{4,5}$ However, only a few of reports on aromatic substitution reactions of the benzene rings of thianthrene and its derivatives have appeared in the literature. ${ }^{6,7}$ Previously, we synthesized 10-monooxy- and 10-dioxy thianthrene-5-sulfilimines and we studied the stereochemistry (cis-trans interconversion) that occurred during acid hydrolysis of the $N$-tosyl group to an secondary amine group as well as the thermal pyramidal inversion on the sulfilimine sulfur. ${ }^{8}$ To clarify the effects of substituents at the peri-position, we also synthesized several 1-substituted thianthrene derivatives and examined their oxidation and $\mathrm{N}$-tosylimination reactions. As a result, we showed that peri-substituents (including hydrogen) have a marked effect on the flip-flop inversion and on reactions on the sulfur atoms. With this background, we were interested in stopping the flip-flop inversion by introducing substituents on the peri-position. The resulting thianthrene derivatives were expected to contribute to the development of a new class of functionalized materials. To elucidate the nature and the reactivity of thianthrene derivatives with fixed flip-flop inversions, we attempted to synthesize thianthrene derivatives bearing substituents on the peri-position. Here we report the synthesis of thianthrene

SYNTHESIS 2014, 46, 0042-0048

Advanced online publication: 07.11.2013

DOI: 10.1055/s-0033-1338550; Art ID: SS-2013-F0493-OP

(c) Georg Thieme Verlag Stuttgart · New York derivatives that use a thianthrene group as a substituent, as a preliminary to fixing the flip-flop inversion.

Thianthrene derivatives bearing substituents in the periposition were prepared by treatment of thianthren-1-yllithium with various electrophilic reagents. Initially, we added butyllithium to a stirred solution of thianthrene (1) in tetrahydrofuran at $-30^{\circ} \mathrm{C}$ under nitrogen. After one hour, the mixture was heated to room temperature and then cooled again to $-30{ }^{\circ} \mathrm{C}$. Various electrophilic reagents were added, and the mixture was stirred for two hours at room temperature under nitrogen to give the corresponding 1-substituted thianthrene derivatives 2a-e (Table 1).

Table 1 Preparation of 1-Substituted Thianthrenes

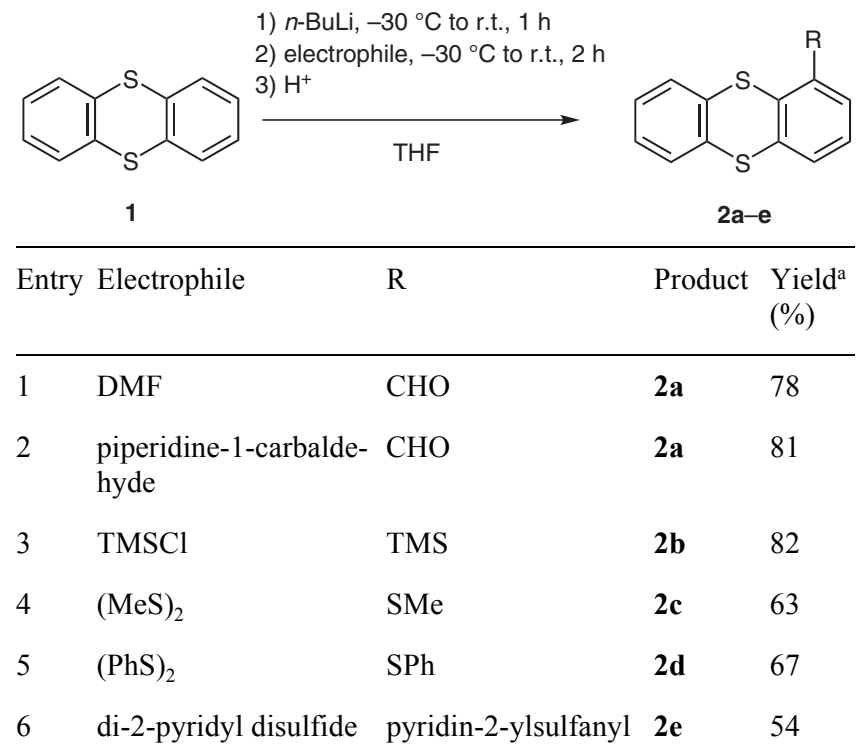

${ }^{a}$ Isolated yield (nonoptimized).

Treatment of aldehyde 2a with thianthren-1-yllithium in tetrahydrofuran under nitrogen gave dithianthren-1-ylmethanol (3) in $81 \%$ yield (Scheme 1).

Oxidation of alcohol $\mathbf{3}$ with oxalyl chloride and dimethyl sulfoxide in the presence of triethylamine gave dithianthren-1-ylmethanone (4) in 84\% yield, whereas treatment of alcohol 3 with thionyl chloride in the presence of pyridine gave 1,1'-(chloromethylene)dithianthrene (5) in $82 \%$ yield (Scheme 2). 

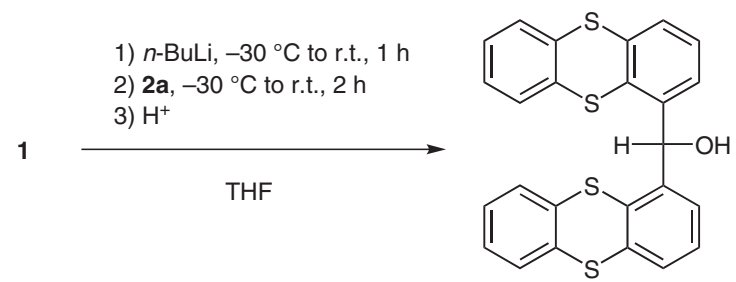

$3(81 \%)$

Scheme 1 Preparation of dithianthren-1-ylmethanol<smiles>O=C(c1cccc2c1Sc1ccccc1S2)c1cccc2c1Sc1ccccc1S2</smiles>

$4(84 \%)$

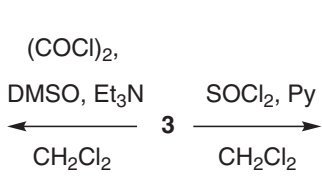<smiles>ClC(c1cccc2c1Sc1ccccc1S2)c1cccc2c1Sc1ccccc1S2</smiles>

$5(82 \%)$
Scheme 2 Reactions of dithianthren-1-ylmethanol (3)

Treatment of chloro compound $\mathbf{5}$ with lithium aluminum hydride gave 1,1'-methylenedithianthrene (6) in $93 \%$ yield (Scheme 3).
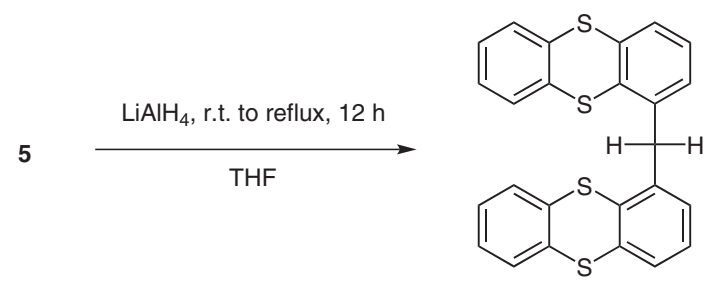

$6(93 \%)$
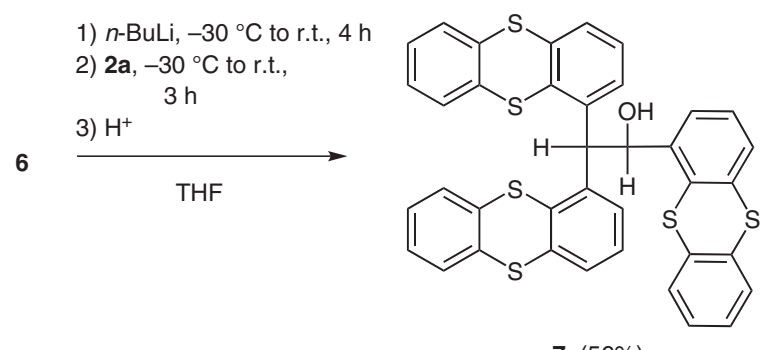

$7(56 \%)$

Scheme 3 Synthesis of 1,1'-methylenedithianthrene (6) and 1,2,2trithianthren-1-ylethanol (7)

These thianthrene derivatives can be regarded as compounds in which the flip-flop inversion is slightly affected by the bulky substituent in the peri-position. A more interesting structure might be obtained by substitution of the thianthrene moiety with a thianthren-1-yl group; however, when 1,1'-methylenedithianthrene (6) was lithiated, the reaction did not take place on the thianthrene framework but occurred, instead, on the methylene part of the mole- cule. Consequently, when lithiated compound $\mathbf{6}$ was allowed to react with aldehyde $2 \mathrm{a}$, we obtained sterically hindered 1,2,2-trithianthren-1-ylethanol (7) in 56\% yield (Scheme 3).

The structures of 6 and 7 were confirmed by single-crystal X-ray crystallographic analysis. ORTEP drawings for $\mathbf{6}$ and 7 are shown in Figures 1 and 2, respectively.

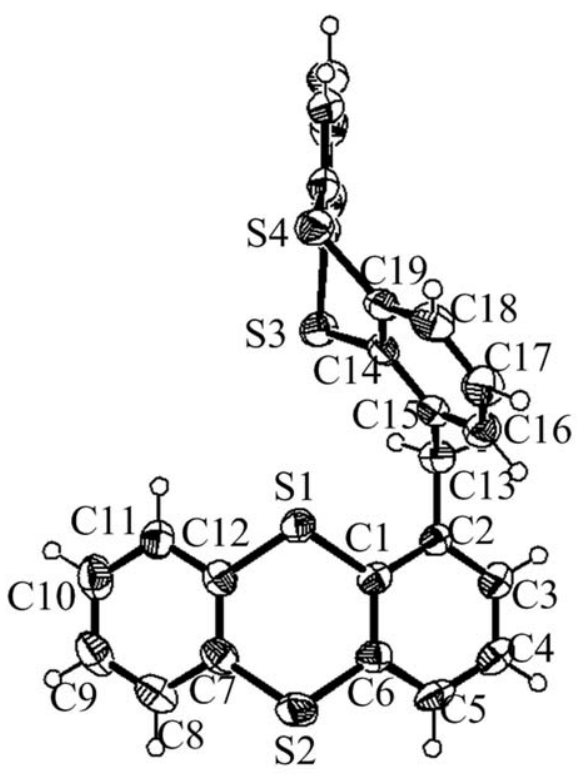

Figure 1 ORTEP drawing of compound 6 showing thermal ellipsoids at the $50 \%$ probability level. Selected bond lengths and angles: $\mathrm{C} 2-\mathrm{C} 13, \quad 1.525(4) \quad \AA ; \quad \mathrm{C} 13-\mathrm{C} 15,1.515(4) \AA \AA$; $\mathrm{C} 2-\mathrm{C} 13-\mathrm{C} 15$, $115.0(2)^{\circ}$.

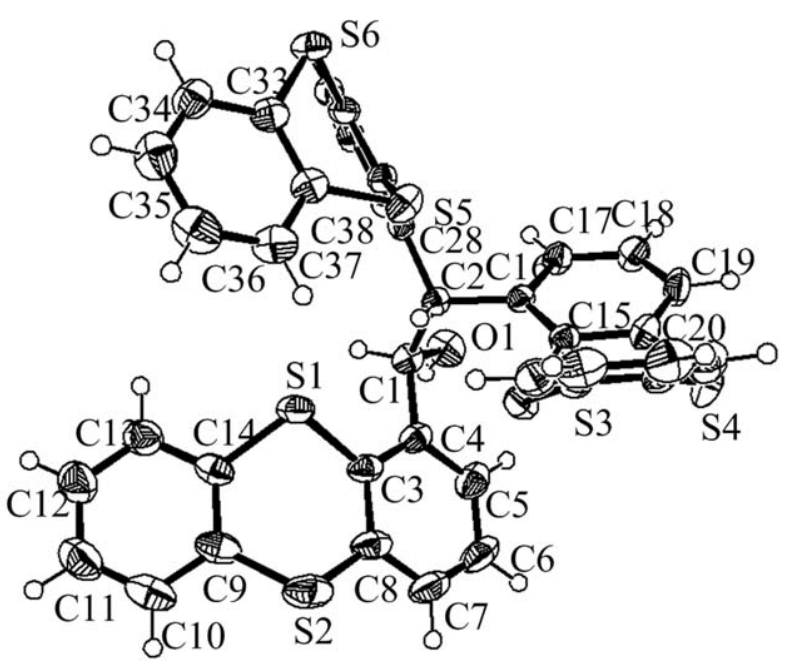

Figure 2 ORTEP drawing of compound 7 showing thermal ellipsoids at the $50 \%$ probability level. Selected bond lengths and angles: $\mathrm{C} 1-\mathrm{C} 2,1.547(5) \AA \AA$; $\mathrm{C} 1-\mathrm{O} 1,1.419(4) \AA \AA ; \mathrm{C} 1-\mathrm{C} 4,1.529(5) \AA$; $\mathrm{C} 2-$ $\mathrm{C} 16,1.527(5) \AA \AA \mathrm{C} 2-\mathrm{C} 28,1.525(5) \AA \AA \mathrm{C} 4-\mathrm{C} 1-\mathrm{O} 1,111.6(3)^{\circ} ; \mathrm{C} 2-$ $\mathrm{C} 1-\mathrm{O} 1,108.3(3)^{\circ}$; $\mathrm{C} 1-\mathrm{C} 2-\mathrm{C} 16,112.2(3)^{\circ}$; $\mathrm{C} 1-\mathrm{C} 2-\mathrm{C} 28,112.8(3)^{\circ}$; $\mathrm{C} 16-\mathrm{C} 2-\mathrm{C} 28,112.9(3)^{\circ}$.

We also attempted to synthesize thianthrene derivatives substituted at the 1- and 9-positions. These syntheses 
could not be carried out in the same way as that used to introduce substituents at the peri-position. To achieve selective reaction at the 1- and 9-positions, thianthrene 5-oxide $(8)^{9-11}$ was dilithiated with lithium diethylamide. Subsequent treatment with chloro(trimethyl)silane at $-30^{\circ} \mathrm{C}$ gave 1,9-bis(trimethylsilyl)thianthrene 5-oxide $(9)^{8,11}$ in $69 \%$ yield. Attempts to introduce substituents other than the trimethylsilyl group onto dilithiated 8 were unsuccessful. We therefore prepared 1,9-dibromothianthrene (10) $)^{8,11}$ by treating sulfoxide 9 with bromine. Dibromo compound $\mathbf{1 0}$ underwent dilithiation with tertbutyllithium ${ }^{11}$ to give the desired dilithiated thianthrene. Moreover, unlike the previous compounds, this could be substituted in the 1- and 9-positions. When we used piperidine-1-carbaldehyde as the electrophile, we obtained thianthrene-1,9-dicarbaldehyde 11 in $84 \%$ yield. Reaction of the dilithiated thianthrene with dialdehyde $\mathbf{1 1}$ as the electrophile gave the macrocyclic diol $\mathbf{1 2}$ in $38 \%$ yield (Scheme 4).

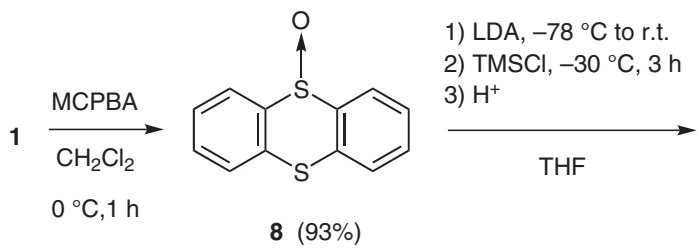<smiles>CS(=O)(=O)c1cccc2c1Sc1cccc(S(C)(=O)=O)c1[SH]2O</smiles>

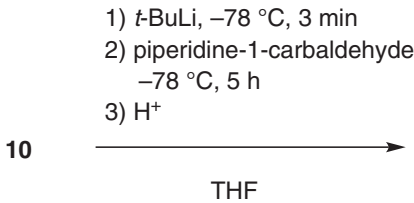<smiles>O=Cc1cccc2c1Sc1ccccc1S2</smiles>
$11(84 \%)$
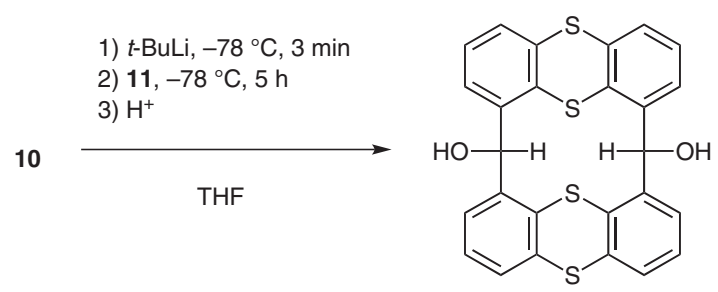

$12(38 \%)$

Scheme 4 Synthesis of 10H,20H-4,6:14,16-diepithiotetrabenzo[b,e,h,k][1,7]dithiacyclododecine-10,20-diol
The structure of 12 was confirmed by single-crystal X-ray crystallography (Figure 3).

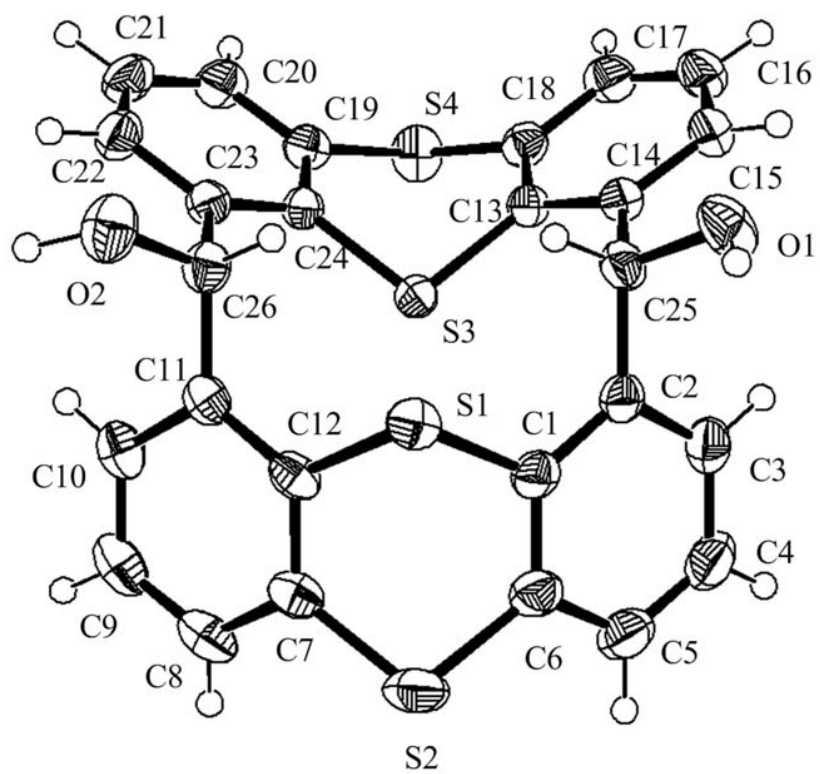

Figure 3 ORTEP drawing of compound 12 showing thermal ellipsoids at the $50 \%$ probability level. Selected bond lengths and angles: $\mathrm{O} 1-\mathrm{C} 25,1.430(3) \AA ; \mathrm{O} 2-\mathrm{C} 26,1.423(2) \AA ;$ C2 $-\mathrm{C} 25,1.521(3) \AA ;$

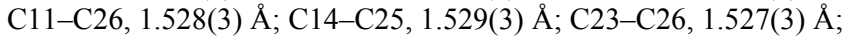
$\mathrm{O} 1-\mathrm{C} 25-\mathrm{C} 2,111.6(2)^{\circ}$; O1-C25-C14, 112.8(2) ; O2-C26-C11, $111.0(2)^{\circ}$; O $2-\mathrm{C} 26-\mathrm{C} 23,107.7(2)^{\circ}$.

Macrocycle $\mathbf{1 2}$ is similar to its acyclic analogue $\mathbf{3}$ in that it is linked by carbon atoms at the peri-positions and the two thianthrene moieties are connected through a single carbon atom; accordingly, it is likely that flip-flop inversion of this interesting compound is greatly hindered. In an attempt to prevent any flip-flop inversion, we examined the synthesis of thianthrene model compounds in which the 1- and 9-positions of the thianthrene moiety were bridged with a chain of carbon atoms.

Initially, we attempted, unsuccessfully, to react dilithiated thianthrene with dibromoalkanes. When dibromomethane or 1,2-dibromoethane was used, dialdehyde 11 and thianthrene were the only products. With $\mathrm{Br}\left(\mathrm{CH}_{2}\right)_{n} \mathrm{Br}(n=3-6)$, we obtained products that were either substituted by bromoalkyl groups in the 1-position only or in both the 1- and 9-positions.

We therefore examined the reactions of disubstituted Grignard reagents $\mathrm{BrMg}\left(\mathrm{CH}_{2}\right)_{n} \mathrm{MgBr}$. The reaction of aldehyde $2 \mathrm{a}$ with $\mathrm{BrMg}\left(\mathrm{CH}_{2}\right)_{4} \mathrm{MgBr}$ gave the expected dimeric product 13 in $63 \%$ yield. In the case of dialdehyde 11, good yields were difficult to achieve, and the bridged diol 14 was obtained in a maximum of $19 \%$ yield (Scheme 5). The reaction mixtures for compounds 12-14 probably contain various diastereomers, but we are unable to separate these. 


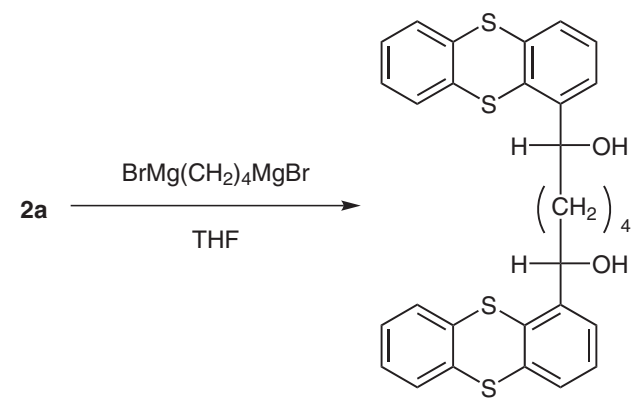

$13(63 \%)$

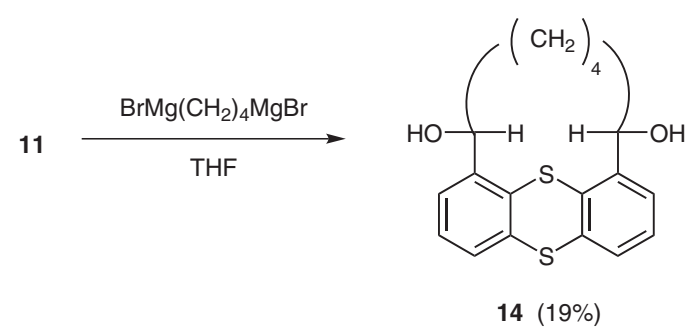

Scheme 5 Reaction of thianthrene carbaldehyde derivatives with disubstituted Grignard reagents

In summary, we have synthesized several monomeric, dimeric, and trimeric thianthrene derivatives linked by carbon atoms. It is possible that the flip-flop inversions of these compounds are affected by the presence of substituents in the peri-positions. The macrocyclic derivatives $\mathbf{1 2}$ and $\mathbf{1 4}$ are of particular interest in this respect. We are currently studying the flip-flop inversions of these compounds, as well their nature and reactivity, and we are attempting to invent a new class of functionalized materials.

All melting points were recorded by using a micro-melting point apparatus and are uncorrected. ${ }^{1} \mathrm{H}$ NMR $(400 \mathrm{MHz})$ and ${ }^{13} \mathrm{C}$ NMR $(100 \mathrm{MHz})$ spectra were recorded on a JEOL JNM-A400 spectrometer in $\mathrm{CDCl}_{3}$ or acetone- $d_{6}$ with TMS as the internal standard. IR spectra were recorded on a Horiba FT-710 spectrophotometer. All reactions were monitored by TLC, and the products were separated by column chromatography on silica gel 60 or by preparative TLC on silica gel $60 \mathrm{PF}_{254}$ with UV or PMA and DNP detection. Mass spectra were recorded on a JEOL JMS-D300 spectrometer. Elemental analyses were performed on a Yanaco JMS-D300 spectrometer. The X-ray crystallographic analyses were performed on Rigaku AF7R four-circle diffractometer by using graphite-monochromated Mo $\mathrm{K} \alpha$ radiation and a rotating anode generator. All reagents were of the highest quality and were further purified by distillation or recrystallization.

\section{Thianthrene-1-ylithium}

A $1.6 \mathrm{M}$ soln of BuLi in THF $(0.35 \mathrm{~mL}, 0.55 \mathrm{mmol})$ was added to a stirred solution of thianthrene $(1 ; 100 \mathrm{mg}, 0.46 \mathrm{mmol})$ in THF $(5$ $\mathrm{mL}$ ) at $-30{ }^{\circ} \mathrm{C}$ under $\mathrm{N}_{2}$. The mixture was refluxed with stirring for $1 \mathrm{~h}$ then cooled at $-30^{\circ} \mathrm{C}$ for used in reactions with electrophiles.

\section{Substituted Thianthrenes 2 and 3; General Reaction ${ }^{12}$}

The electrophile $(0.55 \mathrm{mmol}, 1.2$ equiv) was added to the stirred solution of thianthren-1-yllithium ( $0.46 \mathrm{mmol}, 1.0$ equiv), prepared as described above, under $\mathrm{N}_{2}$ at $-30{ }^{\circ} \mathrm{C}$, and the mixture was stirred for $2 \mathrm{~h}$. The reaction was quenched with $\mathrm{H}_{2} \mathrm{O}(10 \mathrm{~mL})$, and $\mathrm{CHCl}_{3}(20$ $\mathrm{mL}$ ) was added to the mixture. The organic layer was separated, washed successively with $\mathrm{H}_{2} \mathrm{O}(2 \times 20 \mathrm{~mL})$ and brine $(2 \times 20 \mathrm{~mL})$, dried $\left(\mathrm{MgSO}_{4}\right)$, and concentrated under vacuum gave a solid product that was purified by TLC.

Thianthrene-1-carbaldehyde (2a); Typical Reaction ${ }^{12}$

Prepared from piperidine-1-carbaldehyde $(2.4 \mathrm{~mL}, 22 \mathrm{mmol})$ and thianthren-1-yllithium (14.7 mmol), and purified by TLC [silica gel, $\mathrm{CHCl}_{3}$-hexane (1:1)] as a yellow solid; yield: $2.9 \mathrm{~g} \mathrm{(81 \% );} \mathrm{mp} 86-$ $88^{\circ} \mathrm{C}$ (hexane).

IR (KBr): 1670, 1550, 1410, 1370, $1230 \mathrm{~cm}^{-1}$.

${ }^{1} \mathrm{H}$ NMR $\left(400 \mathrm{MHz}, \mathrm{CDCl}_{3}\right): \delta=7.23-7.30(\mathrm{~m}, 2 \mathrm{H}), 7.34(\mathrm{dt}$, $\left.J_{1}=0.4 \mathrm{~Hz}, J_{2}=7.6 \mathrm{~Hz}, 1 \mathrm{H}\right), 7.46-7.49(\mathrm{~m}, 1 \mathrm{H}), 7.50-7.53(\mathrm{~m}, 1$ H), $7.66\left(\mathrm{dd}, J_{1}=1.2 \mathrm{~Hz}, J_{2}=7.6 \mathrm{~Hz}, 1 \mathrm{H}\right), 7.79\left(\mathrm{dd}, J_{1}=1.2 \mathrm{~Hz}\right.$, $\left.J_{2}=7.6 \mathrm{~Hz}, 1 \mathrm{H}\right), 10.54\left(\mathrm{~d}, J_{2}=0.40 \mathrm{~Hz}, 1 \mathrm{H}\right)$.

${ }^{13} \mathrm{C} \mathrm{NMR}\left(100 \mathrm{MHz}, \mathrm{CDCl}_{3}\right): \delta=127.3,128.0,128.4,128.7,128.8$, $129.1,133.6,134.0,134.7,135.5,137.6,140.0,189.9$.

HRMS (EI): $\mathrm{m} / z$ calcd for $\mathrm{C}_{13} \mathrm{H}_{8} \mathrm{OS}_{2}: 244.0017$; found: 244.0009 .

Trimethyl(thianthren-1-yl)silane (2b) ${ }^{9}$

Prepared from TMSCl $(0.06 \mathrm{~mL}, 0.55 \mathrm{mmol})$ and thianthren-1-yllithium $(0.46 \mathrm{mmol})$, and purified by TLC (silica gel, hexane) as a colorless oil; yield: $108.3 \mathrm{mg}(82 \%)$.

IR (neat): 2950, 2880, 1370, $1250 \mathrm{~cm}^{-1}$.

${ }^{1} \mathrm{H}$ NMR $\left(400 \mathrm{MHz}, \mathrm{CDCl}_{3}\right): \delta=0.5(\mathrm{~s}, 9 \mathrm{H}), 7.22-7.30(\mathrm{~m}, 3 \mathrm{H})$, $7.45\left(\mathrm{dd}, J_{1}=7.4 \mathrm{~Hz}, J_{2}=1.2 \mathrm{~Hz}, 1 \mathrm{H}\right), 7.52-7.59(\mathrm{~m}, 3 \mathrm{H})$.

${ }^{13} \mathrm{C}$ NMR $\left(100 \mathrm{MHz}, \mathrm{CDCl}_{3}\right): \delta=-0.1,126.8,127.6,127.7,128.7$, $128.8,129.9,133.9,136.0,136.1,136.7,140.8,142.0$.

\section{1-(Methylsulfanyl)thianthrene (2c)}

Prepared from MeSSMe (52.0 mg, $0.55 \mathrm{mmol})$ and thianthren-1-yllithium $(0.46 \mathrm{mmol})$, and purified by TLC [silica gel, $\mathrm{CHCl}_{3}-$ EtOAc (50:1)] as colorless crystal; yield: $76.0 \mathrm{mg}(63 \%)$; mp 104$105^{\circ} \mathrm{C}$ (hexane).

IR (KBr): 1450, 1430, $1390 \mathrm{~cm}^{-1}$.

${ }^{1} \mathrm{H} \mathrm{NMR}\left(400 \mathrm{MHz}, \mathrm{CDCl}_{3}\right): \delta=2.50(\mathrm{~s}, 3 \mathrm{H}), 7.09-7.13(\mathrm{~m}, 1 \mathrm{H})$, 7.17-7.30 (m, $4 \mathrm{H})$, 7.45-7.49 (m, $1 \mathrm{H}), 7.53-7.58$ (m, $1 \mathrm{H})$.

${ }^{13} \mathrm{C} \mathrm{NMR}\left(100 \mathrm{MHz}, \mathrm{CDCl}_{3}\right): \delta=16.2,124.4,125.4,127.6,127.7$, $127.9,128.5,129.1,133.8,134.9,135.6,135.9,138.9$.

Anal. Calcd for $\mathrm{C}_{13} \mathrm{H}_{10} \mathrm{~S}_{3}$ : C, 59.50; H, 3.84; N, 0.00. Found: C, 59.27; H, 3.84; N, 0.00 .

\section{1-(Phenylsulfanyl)thianthrene (2d)}

Prepared from PhSSPh $(120 \mathrm{mg}, 0.55 \mathrm{mmol})$ and thianthren-1-yllithium $(0.46 \mathrm{mmol})$, and purified by TLC [silica gel, hexane$\left.\mathrm{CHCl}_{3}(1: 10)\right]$ as colorless crystals; yield: $99.8 \mathrm{mg}(67 \%)$; mp 50$51{ }^{\circ} \mathrm{C}$ (hexane).

IR (KBr): 1447, 1428, $1392 \mathrm{~cm}^{-1}$.

${ }^{1} \mathrm{H}$ NMR $\left(400 \mathrm{MHz}, \mathrm{CDCl}_{3}\right): \delta=7.03\left(\mathrm{dd}, J_{1}=1.2 \mathrm{~Hz}, J_{2}=4.6 \mathrm{~Hz}\right.$, $1 \mathrm{H}), 6.97-7.01(\mathrm{~m}, 1 \mathrm{H}), 7.13-7.25(\mathrm{~m}, 4 \mathrm{H}), 7.21-7.31(\mathrm{~m}, 1 \mathrm{H})$, $7.39-7.44(\mathrm{~m}, 2 \mathrm{H}), 7.50\left(\mathrm{dd}, J_{1}=0.8 \mathrm{~Hz}, J_{2}=7.8 \mathrm{~Hz}, 1 \mathrm{H}\right), 7.55$ $\left(\mathrm{dd}, J_{1}=0.8 \mathrm{~Hz}, J_{2}=7.6 \mathrm{~Hz}, 1 \mathrm{H}\right), 8.41(\mathrm{~d}, J=3.4 \mathrm{~Hz}, 1 \mathrm{H})$.

${ }^{13} \mathrm{C} \mathrm{NMR}\left(100 \mathrm{MHz}, \mathrm{CDCl}_{3}\right): \delta=120.1,121.4,127.7,127.8,128.2$, 128.8, 129.8, 130.6, 134.8, 135.1, 135.4, 136.4, 136.7, 141.7, 149.6, 159.3 .

Anal. Calcd for $\mathrm{C}_{18} \mathrm{H}_{12} \mathrm{~S}_{3}$ : C, 66.63; H, 3.72; N, 0.00. Found: C, $66.38 ; \mathrm{H}, 3.70 ; \mathrm{N}, 0.00$.

\section{2-(Thianthren-1-ylsulfanyl)pyridine (2e)}

Prepared from di-2-pyridyl disulfide $(121.5 \mathrm{mg}, 0.55 \mathrm{mmol})$ and thianthren-1-yllithium ( $0.46 \mathrm{mmol})$, and purified by TLC [silica gel, EtOAc-hexane (5:1)] as a yellow solid; yield: $80.5 \mathrm{mg} \mathrm{(54 \% );} \mathrm{mp}$ $72-73{ }^{\circ} \mathrm{C}$ (hexane).

IR (KBr): 3050, 1570, 1280, 1150, 1120, 980, 750, $710 \mathrm{~cm}^{-1}$. 
${ }^{1} \mathrm{H} \mathrm{NMR}\left(400 \mathrm{MHz}, \mathrm{CDCl}_{3}\right): \delta=6.87\left(\mathrm{dd}, J_{1}=0.8 \mathrm{~Hz}, J_{2}=8.2 \mathrm{~Hz}\right.$, $1 \mathrm{H}), 7.00-7.04(\mathrm{~m}, 1 \mathrm{H}), 7.17-7.28(\mathrm{~m}, 3 \mathrm{H}), 7.34\left(\mathrm{dd}, J_{1}=1.2 \mathrm{~Hz}\right.$ $\left.J_{2}=7.6 \mathrm{~Hz}, 1 \mathrm{H}\right), 7.43-7.49(\mathrm{~m}, 2 \mathrm{H}), 7.54\left(\mathrm{dd}, J_{1}=1.2 \mathrm{~Hz}, J_{2}=7.8\right.$ $\mathrm{Hz}, 1 \mathrm{H}), 7.59$ (dd, $\left.J_{1}=1.2 \mathrm{~Hz}, J_{2}=8.8 \mathrm{~Hz}, 1 \mathrm{H}\right), 8.42-8.45(\mathrm{~m}, 1$ $\mathrm{H})$.

${ }^{13} \mathrm{C} \mathrm{NMR}\left(100 \mathrm{MHz}, \mathrm{CDCl}_{3}\right): \delta=120.2,121.5,127.8,127.9,128.0$, $128.3,128.9,129.9,130.8,134.9,135.3,135.6,136.6,136.8,141.9$, $149.8,159.5$.

HRMS (EI): $m / z$ calcd for $\mathrm{C}_{17} \mathrm{H}_{11} \mathrm{NS}_{3}: 325.0054$; found: 325.0050 .

\section{Dithianthren-1-ylmethanol (3)}

Prepared from aldehyde $\mathbf{2 b}(677 \mathrm{mg}, 2.77 \mathrm{mmol})$ and thianthren-1yllithium (4.16 mmol), and purified by TLC [silica gel, EtOAchexane (1:4)] to give colorless crystals; yield: $1.03 \mathrm{~g} \mathrm{(81 \% );} \mathrm{mp}$ 200-201 ${ }^{\circ} \mathrm{C}\left(\mathrm{CHCl}_{3}\right.$-hexane).

${ }^{1} \mathrm{H}$ NMR (400 MHz, $\left.\mathrm{CDCl}_{3}\right): \delta=6.85(\mathrm{~s}, 1 \mathrm{H}), 7.18-7.28(\mathrm{~m}, 8 \mathrm{H})$, 7.42-7.51 (m, $6 \mathrm{H})$.

${ }^{13} \mathrm{C} \mathrm{NMR}\left(100 \mathrm{MHz}, \mathrm{CDCl}_{3}\right): \delta=71.3,126.5,127.5,127.7,127.9$, $128.5,128.6,129.1,135.0,135.1,136.5,136.6,141.7$.

IR (KBr): 3367, 2955, 2929, 2858, 1447, 1409, $749 \mathrm{~cm}^{-1}$.

Anal. Calcd for $\mathrm{C}_{25} \mathrm{H}_{16} \mathrm{OS}_{4}: \mathrm{C}, 65.18 ; \mathrm{H}, 3.50 ; \mathrm{N}, 0.00$. Found: $\mathrm{C}$, $65.10 ; \mathrm{H}, 3.55 ; \mathrm{N}, 0.00$.

\section{Dithianthren-1-ylmethanone (4)}

Oxalyl chloride ( $622 \mathrm{mg}, 4.90 \mathrm{mmol}$ ) was added to a stirred solution of alcohol $3(500 \mathrm{mg}, 1.09 \mathrm{mmol})$ in $\mathrm{CH}_{2} \mathrm{Cl}_{2}(18 \mathrm{~mL})$ at $-78^{\circ} \mathrm{C}$ un$\operatorname{der} \mathrm{N}_{2}$, and the mixture was stirred for $15 \mathrm{~min}$. DMSO (340.6 mg, $4.36 \mathrm{mmol})$ was added followed, after $30 \mathrm{~min}$, by $\mathrm{Et}_{3} \mathrm{~N}(608 \mu \mathrm{L}$, $4.36 \mathrm{mmol}$ ), and the mixture was stirred for $1 \mathrm{~h}$. The mixture was then washed with $\mathrm{H}_{2} \mathrm{O}(2 \times 30 \mathrm{~mL})$, and extracted with $\mathrm{CHCl}_{3}(2 \times$ $40 \mathrm{~mL}$ ). The organic layer was separated, washed successively with $\mathrm{H}_{2} \mathrm{O}(2 \times 20 \mathrm{~mL})$ and brine $(2 \times 20 \mathrm{~mL})$, dried $\left(\mathrm{MgSO}_{4}\right)$, and concentrated under vacuum. The solid product was purified by crystallization (EtOAc) to give yellow crystals; yield: $424.9 \mathrm{mg}$ (84\%); mp $232{ }^{\circ} \mathrm{C}($ EtOAc $)$.

IR (KBr): 3055, 1645, 1396, 1290, $1255 \mathrm{~cm}^{-1}$.

${ }^{1} \mathrm{H}$ NMR $\left(400 \mathrm{MHz}, \mathrm{CDCl}_{3}\right): \delta=7.17-7.31(\mathrm{~m}, 8 \mathrm{H}), 7.35(\mathrm{~d}$, $J=7.6 \mathrm{~Hz}, 2 \mathrm{H}), 7.47(\mathrm{~d}, J=8.0 \mathrm{~Hz}, 2 \mathrm{H}), 7.65(\mathrm{~d}, J=7.6 \mathrm{~Hz}, 2 \mathrm{H})$.

${ }^{13} \mathrm{C} \mathrm{NMR}\left(100 \mathrm{MHz}, \mathrm{CDCl}_{3}\right): \delta=126.3,127.8,128.0,128.5,129.0$, $129.9,132.0,135.3,136.0,136.9,138.5,138.7,194.5$.

Anal. Calcd for $\mathrm{C}_{25} \mathrm{H}_{14} \mathrm{OS}_{4}$ : C, 65.47; H, 3.08; N, 0.00. Found: C, $65.51 ; \mathrm{H}, 3.16 ; \mathrm{N}, 0.00$.

\section{1,1'-(Chloromethylene)dithianthrene (5)}

Pyridine $(52.6 \mu \mathrm{L}, 0.65 \mathrm{mmol})$ was added to a stirred solution of alcohol $3(150 \mathrm{mg}, 0.65 \mathrm{mmol})$ in $\mathrm{CHCl}_{3}(16 \mathrm{~mL})$ at r.t. under $\mathrm{N}_{2}$, and the mixture was stirred for $10 \mathrm{~min}$. $\mathrm{SOCl}_{2}(59 \mu \mathrm{L}, 0.81 \mathrm{mmol})$ was added and, after $30 \mathrm{~min}$, the mixture was refluxed for $2 \mathrm{~h}$. The solution was washed with $\mathrm{H}_{2} \mathrm{O}(2 \times 20 \mathrm{~mL})$, and extracted with $\mathrm{CHCl}_{3}$ $(2 \times 30 \mathrm{~mL})$. The organic layer was separated, washed successively with $\mathrm{H}_{2} \mathrm{O}(2 \times 25 \mathrm{~mL})$ and brine $(2 \times 25 \mathrm{~mL})$, dried $\left(\mathrm{MgSO}_{4}\right)$, and concentrated under vacuum. The solid product was purified by crystallization $\left(\mathrm{CH}_{2} \mathrm{Cl}_{2}\right.$-hexane) to give colorless crystals; yield: 127.6 $\mathrm{mg}(82 \%)$; $\mathrm{mp} 219^{\circ} \mathrm{C}\left(\mathrm{CH}_{2} \mathrm{Cl}_{2}\right.$-hexane).

IR (KBr): $1444 \mathrm{~cm}^{-1}$.

${ }^{1} \mathrm{H}$ NMR $\left(400 \mathrm{MHz}, \mathrm{CDCl}_{3}\right): \delta=7.22-7.28(\mathrm{~m}, 6 \mathrm{H}), 7.37(\mathrm{~s}, 1 \mathrm{H})$, 7.40-7.52 (m, $8 \mathrm{H})$

${ }^{13} \mathrm{C}$ NMR $\left(100 \mathrm{MHz}, \mathrm{CDCl}_{3}\right): \delta=59.1,127.5,127.7,127.8,128.0$, $128.7,128.8,129.2,134.9,135.2,136.5,137.2,139.7$.

Anal. Calcd for $\mathrm{C}_{25} \mathrm{H}_{15} \mathrm{ClS}_{4}$ : C, 62.67; H, 3.16; N, 0.00. Found: C, $62.62 ; \mathrm{H}, 3.18 ; \mathrm{N}, 0.00$.

\section{1,1'-Methylenedithianthrene (6)}

A solution of $\mathrm{LiAlH}_{4}(35.5 \mathrm{mg}, 0.94 \mathrm{mmol})$ in THF $(10 \mathrm{~mL})$ was added to a stirred solution of chloro compound 5 (90 mg, 0.19 $\mathrm{mmol})$ in THF $(9 \mathrm{~mL})$ at r.t. under $\mathrm{N}_{2}$. The mixture was refluxed for $12 \mathrm{~h}$ and then the reaction was quenched with $\mathrm{H}_{2} \mathrm{O}(8 \mathrm{~mL}) . \mathrm{CHCl}_{3}$ $(25 \mathrm{~mL})$ was added, and the organic layer was separated, washed successively with $\mathrm{H}_{2} \mathrm{O}(2 \times 20 \mathrm{~mL})$ and brine $(2 \times 20 \mathrm{~mL})$, dried $\left(\mathrm{MgSO}_{4}\right)$, and concentrated under vacuum. The solid product was purified by TLC [silica gel, EtOAc-hexane (5:1)] to give colorless crystals; yield: $77 \mathrm{mg}$ (93\%); mp $140-141{ }^{\circ} \mathrm{C}$ (from $\mathrm{CH}_{2} \mathrm{Cl}_{2}-$ hexane).

IR (KBr): $1441,1400 \mathrm{~cm}^{-1}$

${ }^{1} \mathrm{H}$ NMR $\left(400 \mathrm{MHz}, \mathrm{CDCl}_{3}\right): \delta=4.48(\mathrm{~s}, 2 \mathrm{H}), 6.95\left(\mathrm{dd}, J_{1}=0.8\right.$ $\left.\mathrm{Hz}, J_{2}=7.4 \mathrm{~Hz}, 2 \mathrm{H}\right), 7.14(\mathrm{t}, J=7.4 \mathrm{~Hz}, 2 \mathrm{H}), 7.21-7.26(\mathrm{~m}, 4 \mathrm{H})$, 7.40-7.52 (m, $6 \mathrm{H})$.

${ }^{13} \mathrm{C}$ NMR $\left(100 \mathrm{MHz}, \mathrm{CDCl}_{3}\right): \delta=38.9,127.3,127.4,127.6,127.8$, $128.6,128.8,129.1,135.4,135.8,136.0,136.3,139.1$.

HRMS (EI): $m / z$ calcd for $\mathrm{C}_{25} \mathrm{H}_{16} \mathrm{~S}_{4}: 444.0135$; found: 444.0141 .

X-ray crystal data: ${ }^{13}$ Empirical formula: $\mathrm{C}_{25} \mathrm{H}_{16} \mathrm{~S}_{4}$; Formula weight: 444.64; Crystal system = triclinic; Space group P $\overline{1}(\# 2)$; Lattice parameters: $a=11.181(5) \AA ; b=11.821(9) \AA, c=8.679(3) \AA$; $\alpha=94.78(3)^{\circ} ; \quad \mathrm{V}=1023.2(10) \quad \AA^{3} ; \quad \mathrm{T}=23.0{ }^{\circ} \mathrm{C} ; \quad \mathrm{Z}=4 ; \quad \mu$ $(\mathrm{MoK} \alpha)=9.48 \mathrm{~cm}^{-1} ; 6255$ reflections measured, 5973 unique $\left(R_{\text {int }}=0.048\right)$; final $R$ value $=0.094$.

\section{1,2,2-Trithianthren-1-ylethanol (7)}

A 1.6 M soln of BuLi in THF $(0.18 \mathrm{~mL}, 0.29 \mathrm{mmol})$ was added to a stirred solution of 1,1'-methylenedithianthrene $(6 ; 100 \mathrm{mg}, 0.23$ $\mathrm{mmol})$ in THF $(10 \mathrm{~mL})$ under $\mathrm{N}_{2}$ at $-50^{\circ} \mathrm{C}$. After $3 \mathrm{~h}$, the mixture was warmed to r.t. and stirred for $1 \mathrm{~h}$, then cooled to $-30^{\circ} \mathrm{C}$. A solution of aldehyde $2 \mathrm{a}(110 \mathrm{mg}, 0.45 \mathrm{mmol})$ in THF $(5 \mathrm{~mL})$ was added to the mixture, which was stirred for $3 \mathrm{~h}$. The reaction was quenched with $\mathrm{H}_{2} \mathrm{O}(8 \mathrm{~mL})$, and the product was extracted with $\mathrm{CHCl}_{3}(2 \times 25 \mathrm{~mL})$. The organic layers were combined, washed successively with $\mathrm{H}_{2} \mathrm{O}(2 \times 20 \mathrm{~mL})$ and brine $(2 \times 20 \mathrm{~mL})$, dried $\left(\mathrm{MgSO}_{4}\right)$, and concentrated under vacuum gave a solid product. The product was purified by TLC [silica gel, $\mathrm{CHCl}_{3}$-hexane $(2: 1)$ ] to give colorless crystals; yield: $87.2 \mathrm{mg}(56 \%)$; mp $261-262^{\circ} \mathrm{C}$ $\left(\mathrm{CH}_{2} \mathrm{Cl}_{2}\right.$-hexane).

IR (KBr): 3462, 1442, $1408 \mathrm{~cm}^{-1}$.

${ }^{1} \mathrm{H}$ NMR $\left(400 \mathrm{MHz}, \mathrm{CDCl}_{3}\right): \delta=5.98(\mathrm{~d}, J=5.2 \mathrm{~Hz}, 1 \mathrm{H}), 6.08(\mathrm{~d}$, $J=4.8 \mathrm{~Hz}, 1 \mathrm{H}), 6.75\left(\mathrm{dd}, J_{1}=1.2 \mathrm{~Hz}, J_{2}=7.6 \mathrm{~Hz}, 1 \mathrm{H}\right), 6.95-7.20$ $(\mathrm{m}, 8 \mathrm{H}), 7.22-7.33(\mathrm{~m}, 4 \mathrm{H}), 7.37-7.40(\mathrm{~m}, 4 \mathrm{H}), 7.43\left(\mathrm{dd}, J_{1}=1.2\right.$ $\left.\mathrm{Hz}, J_{2}=7.6 \mathrm{~Hz}, 1 \mathrm{H}\right), 7.52-7.61(\mathrm{~m}, 2 \mathrm{H}), 7.94(\mathrm{~d}, J=7.6 \mathrm{~Hz}, 1 \mathrm{H})$;

${ }^{13} \mathrm{C}$ NMR $\left(100 \mathrm{MHz}, \mathrm{CDCl}_{3}\right): \delta=50.4,73.6,126.1,126.9,127.2$, 127.2, 127.3, 127.4, 127.6, 127.6, 127.7, 127.8, 128.0, 128.2, 128.8, $128.9,129.3,129.4,129.5,134.6,135.2,136.3,136.4,136.4,136.5$, $136.6,136.7,136.7,136.8,138.3,138.4,141.5,141.9$.

HRMS (EI): $m / z$ calcd for $\mathrm{C}_{38} \mathrm{H}_{24} \mathrm{OS}_{6}$ : 688.0151; found: 688.0147 .

X-ray crystal data: ${ }^{13}$ Empirical formula: $\mathrm{C}_{38} \mathrm{H}_{24} \mathrm{OS}_{6}\left(\mathrm{CH}_{2} \mathrm{Cl}_{2}\right)$; Formula weight 773.90; Crystal system $=$ triclinic; Space group P $\overline{1}$ (\#2); Lattice parameters: $a=11.868(2) \AA ; \quad b=16.470(2) \AA$, $c=9.280(2) \AA ; \alpha=98.37(1)^{\circ} ; \mathrm{V}=1716.8(5) \AA^{3} ; \mathrm{T}=-20.0{ }^{\circ} \mathrm{C}$; $\mathrm{Z}=2 ; \mu(\mathrm{MoK} \alpha)=5.87 \mathrm{~cm}^{-1} ; 10428$ reflections measured, 6378 unique $\left(R_{\text {int }}=0.062\right)$; final $R$ value 0.062 .

\section{Thianthrene 5-Oxide $(8)^{9,11}$}

A soln of MCPBA (878 mg, $5.08 \mathrm{mmol})$ in $\mathrm{CH}_{2} \mathrm{Cl}_{2}(15 \mathrm{~mL})$ was added to a stirred solution of thianthrene $(1 ; 1.0 \mathrm{~g}, 4.62 \mathrm{mmol})$ in $\mathrm{CH}_{2} \mathrm{Cl}_{2}(15 \mathrm{~mL})$ cooled in an ice bath under $\mathrm{N}_{2}$. After $1 \mathrm{~h}$, the mixture was extracted with sat. aq $\mathrm{NaHCO}_{3}(3 \times 15 \mathrm{~mL})$. The organic layer was washed with $\mathrm{H}_{2} \mathrm{O}(2 \times 25 \mathrm{~mL})$ then dried $\left(\mathrm{MgSO}_{4}\right)$ and concentrated under vacuum. The solid product was purified by TLC [silica gel, EtOAc-hexane (1:1)] to give colorless crystals; yield: $998 \mathrm{mg}$ (93\%); mp $143-144{ }^{\circ} \mathrm{C}\left(\mathrm{CH}_{2} \mathrm{Cl}_{2}\right.$-hexane).

IR (KBr): 1430, 1120, 1075, $1030 \mathrm{~cm}^{-1}$.

${ }^{1} \mathrm{H}$ NMR $\left(400 \mathrm{MHz}, \mathrm{CDCl}_{3}\right): \delta=7.41-7.46(\mathrm{~m}, 2 \mathrm{H}), 7.54-7.58(\mathrm{~m}$, $2 \mathrm{H}), 7.62-7.65(\mathrm{~m}, 2 \mathrm{H}), 7.93\left(\mathrm{dd}, J_{1}=1.2 \mathrm{~Hz}, J_{2}=6.8 \mathrm{~Hz}, 2 \mathrm{H}\right)$. 
${ }^{13} \mathrm{C} \mathrm{NMR}\left(100 \mathrm{MHz}, \mathrm{CDCl}_{3}\right): \delta=124.2,128.1,128.2,128.7,129.6$, 140.8 .

HRMS (EI): $m / z$ calcd for $\mathrm{C}_{12} \mathrm{H}_{8} \mathrm{OS}_{2}: 232.0017$; found: 232.0013 .

\section{1,9-Bis(trimethylsilyl)thianthrene 5-Oxide (9) $)^{9,11}$}

A $1.8 \mathrm{M}$ soln of LDA in THF $(6.0 \mathrm{~mL}, 10.8 \mathrm{mmol})$ was added to a stirred solution of oxide $8(1.0 \mathrm{~g}, 4.30 \mathrm{mmol})$ in THF $(25 \mathrm{~mL})$ at $-78{ }^{\circ} \mathrm{C}$ under $\mathrm{N}_{2}$. After $3 \mathrm{~h}$, the mixture was warmed to r.t., stirred for $30 \mathrm{~min}$, and then cooled to $-30^{\circ} \mathrm{C}$. TMSCl $(1.41 \mathrm{~g}, 13.0 \mathrm{mmol})$ was added, and the mixture was stirred for $3 \mathrm{~h}$. The reaction was quenched with $\mathrm{H}_{2} \mathrm{O}(15 \mathrm{~mL})$, and $\mathrm{CHCl}_{3}(30 \mathrm{~mL})$ was added. The organic layer was separated, washed successively with $\mathrm{H}_{2} \mathrm{O}(2 \times 30$ $\mathrm{mL})$ and brine $(2 \times 20 \mathrm{~mL})$, dried $\left(\mathrm{MgSO}_{4}\right)$, and concentrated under vacuum to give a solid product. The product was purified by TLC [silica gel, EtOAc-hexane (1:3)] to give colorless crystals; yield: $1.04 \mathrm{~g}(64 \%)$; mp 213-214 ${ }^{\circ} \mathrm{C}$ (hexane).

IR (KBr): 2952, 1547, 1364, 1247, 1023, 845, 786, $760 \mathrm{~cm}^{-1}$.

${ }^{1} \mathrm{H} \mathrm{NMR}\left(400 \mathrm{MHz}, \mathrm{CDCl}_{3}\right): \delta=0.56(\mathrm{~s}, 18 \mathrm{H}), 7.44(\mathrm{t}, J=8.0 \mathrm{~Hz}$, $2 \mathrm{H}), 7.63\left(\mathrm{dd}, J_{1}=1.2 \mathrm{~Hz}, J_{2}=7.2 \mathrm{~Hz}, 2 \mathrm{H}\right), 7.73\left(\mathrm{dd}, J_{1}=1.2 \mathrm{~Hz}\right.$, $\left.J_{2}=7.6 \mathrm{~Hz}, 2 \mathrm{H}\right)$.

${ }^{13} \mathrm{C} \mathrm{NMR}\left(100 \mathrm{MHz}, \mathrm{CDCl}_{3}\right): \delta=1.4,129.5,130.1,134.0,134.2$, 141.0, 144.4 .

\section{1,9-Dibromothianthrene (10) $)^{9,11}$}

$\mathrm{Br}_{2}(895 \mathrm{mg}, 5.60 \mathrm{mmol})$ was added to a stirred solution of disilylated derivative $9(300 \mathrm{mg}, 0.80 \mathrm{mmol})$ in $\mathrm{CCl}_{4}(15 \mathrm{~mL})$ at r.t. under $\mathrm{N}_{2}$. The mixture was stirred for $10 \mathrm{~h}$ and then the reaction was quenched with aq $1 \mathrm{M} \mathrm{Na}_{2} \mathrm{~S}_{2} \mathrm{O}_{3}(5 \mathrm{~mL})$, and the mixture was washed with $\mathrm{H}_{2} \mathrm{O}(2 \times 20 \mathrm{~mL})$, and extracted with $\mathrm{CHCl}_{3}(2 \times 30 \mathrm{~mL})$. The organic layers were combined, washed successively with $\mathrm{H}_{2} \mathrm{O}(2 \times$ $20 \mathrm{~mL})$ and brine $(2 \times 20 \mathrm{~mL})$, dried $\left(\mathrm{MgSO}_{4}\right)$, and concentrated under vacuum gave a solid product. The product was purified by crystallization (toluene) to give colorless crystals; yield: $292 \mathrm{mg}(98 \%)$; mp 213-214 ${ }^{\circ} \mathrm{C}$ (toluene).

${ }^{1} \mathrm{H} \mathrm{NMR}\left(400 \mathrm{MHz}, \mathrm{CDCl}_{3}\right): \delta=7.10(\mathrm{t}, J=8.0 \mathrm{~Hz}, 2 \mathrm{H}), 7.42(\mathrm{dd}$, $\left.J_{1}=1.2 \mathrm{~Hz}, J_{2}=7.8 \mathrm{~Hz}, 2 \mathrm{H}\right), 7.53\left(\mathrm{dd}, J_{1}=1.2 \mathrm{~Hz}, J_{2}=7.8 \mathrm{~Hz}, 2\right.$ $\mathrm{H})$.

${ }^{13} \mathrm{C} \mathrm{NMR}\left(100 \mathrm{MHz}, \mathrm{CDCl}_{3}\right): \delta=123.3,127.4,128.7,131.9,136.4$, 136.6.

HRMS (EI): $m / z$ calcd for $\mathrm{C}_{12} \mathrm{H}_{6} \mathrm{Br}_{2} \mathrm{~S}_{2}: 371.8278$; found: 371.8271 .

\section{Thianthrene-1,9-dicarbaldehyde (11)}

A $1.7 \mathrm{M}$ soln of $t$-BuLi in THF (1.89 mL, $3.21 \mathrm{mmol})$ was added to a stirred solution of dibromo compound $10(200 \mathrm{mg}, 0.53 \mathrm{mmol})$ in THF $(20 \mathrm{~mL})$ at $-78{ }^{\circ} \mathrm{C}$ under $\mathrm{N}_{2}$. After $3 \mathrm{~min}$, piperidine-1-carbaldehyde (164 $\mathrm{mg}, 1.45 \mathrm{mmol}$ ) was added, and the mixture was stirred for $5 \mathrm{~h}$. The reaction as then quenched with $\mathrm{H}_{2} \mathrm{O}(15 \mathrm{~mL})$, and the mixture was extracted with $\mathrm{CHCl}_{3}(2 \times 40 \mathrm{~mL})$. The organic layers were combined, washed with $\mathrm{H}_{2} \mathrm{O}(3 \times 40 \mathrm{~mL})$, dried $\left(\mathrm{MgSO}_{4}\right)$, and concentrated under reduced pressure to give a crude product. The product was purified by crystallization $\left(\mathrm{CH}_{2} \mathrm{Cl}_{2}\right.$-hexane $)$ to give yellow crystals; yield: $108 \mathrm{mg}(84 \%)$; mp $226-228^{\circ} \mathrm{C}\left(\mathrm{CH}_{2} \mathrm{Cl}_{2}-\right.$ hexane).

IR (KBr): 1685, $1232 \mathrm{~cm}^{-1}$.

${ }^{1} \mathrm{H} \mathrm{NMR}\left(400 \mathrm{MHz}, \mathrm{CDCl}_{3}\right): \delta=7.46\left(\mathrm{dt}, J_{1}=0.8 \mathrm{~Hz}, J_{2}=7.6 \mathrm{~Hz}\right.$, $2 \mathrm{H}), 7.76\left(\mathrm{dd}, J_{1}=1.2 \mathrm{~Hz}, J_{2}=7.6 \mathrm{~Hz}, 2 \mathrm{H}\right), 7.89\left(\mathrm{dd}, J_{1}=1.6 \mathrm{~Hz}\right.$, $\left.J_{2}=7.6 \mathrm{~Hz}, 2 \mathrm{H}\right), 10.64(\mathrm{~s}, 2 \mathrm{H})$.

${ }^{13} \mathrm{C} \mathrm{NMR}\left(100 \mathrm{MHz}, \mathrm{CDCl}_{3}\right): \delta=128.3,128.9,133.8,135.4,138.1$, 138.3, 189.4 .

Anal. Calcd for $\mathrm{C}_{14} \mathrm{H}_{8} \mathrm{O}_{2} \mathrm{~S}_{2}:$ C, 61.74; H, 2.96; N, 0.00; found: C, 61.40; H, 3.16; N, 0.00 .

$10 H, 20 H-4,6: 14,16-D i e p i t h i o t e t r a b e n z o[b, e, h, k][1,7]$ dithiacyclododecine-10,20-diol (12)

A $1.7 \mathrm{M}$ soln of $t$-BuLi in THF $(0.26 \mathrm{~mL}, 0.44 \mathrm{mmol})$ was added to a stirred solution of dibromo compound $\mathbf{1 0}(27.5 \mathrm{mg}, 0.07 \mathrm{mmol})$ in
THF $(14.7 \mathrm{~mL})$ at $-78^{\circ} \mathrm{C}$ under $\mathrm{N}_{2}$. After $3 \mathrm{~min}$, a soln of dialdehyde $11(20 \mathrm{mg}, 0.07 \mathrm{mmol})$ in THF $(5 \mathrm{~mL})$ was added, and the mixture and stirred for $5 \mathrm{~h}$. The reaction was then quenched with $\mathrm{H}_{2} \mathrm{O}(5 \mathrm{~mL})$, and $\mathrm{CHCl}_{3}(2 \times 20 \mathrm{~mL})$ was added to the mixture. The organic layer was separated, washed successively with $\mathrm{H}_{2} \mathrm{O}(2 \times 20$ $\mathrm{mL})$ and brine $(2 \times 20 \mathrm{~mL})$, dried $\left(\mathrm{MgSO}_{4}\right)$, and concentrated under vacuum to give a solid product. The product was purified by TLC [silica gel, EtOAc-hexane (1:2)] to give colorless crystals; yield: $12.9 \mathrm{mg}(38 \%) ; \mathrm{mp} 343-345^{\circ} \mathrm{C}\left(\mathrm{CHCl}_{3}\right.$-EtOAc-hexane).

IR (KBr): 3413 (br), 1402, $1032 \mathrm{~cm}^{-1}$.

${ }^{1} \mathrm{H}$ NMR $\left(400 \mathrm{MHz}, \mathrm{CDCl}_{3}\right): \delta=7.16(\mathrm{~s}, 2 \mathrm{H}), 7.21(\mathrm{t}, J=7.8 \mathrm{~Hz}$, $4 \mathrm{H}), 7.40(\mathrm{~d}, J=7.6 \mathrm{~Hz}, 4 \mathrm{H}), 7.52(\mathrm{~d}, J=7.2 \mathrm{~Hz}, 4 \mathrm{H})$.

${ }^{13} \mathrm{C}$ NMR (100 MHz, acetone- $\left.d_{6}\right): \delta=72.1,127.7,126.0,127.9$, $128.1,134.1,138.9,144.4$.

HRMS (EI): $m / z$ calcd for $\mathrm{C}_{14} \mathrm{H}_{12} \mathrm{OS}_{2}: 488.0033$; found: 488.0028 .

X-ray crystal data: ${ }^{13}$ Empirical formula: $\mathrm{C}_{26} \mathrm{H}_{16} \mathrm{O}_{2} \mathrm{~S}_{4} \cdot\left(\mathrm{C}_{4} \mathrm{H}_{8} \mathrm{O}_{2}\right)$; Formula weight 576.76; Crystal system $=$ triclinic; Space group $\mathrm{P} \overline{1}$ (\#2); Lattice parameters: $a=10.767(1) \AA ; \quad b=11.785(1) \AA$, $c=10.699(1) \AA ; \alpha=100.840(10)^{\circ}, \beta=90.851(9)^{\circ}, \gamma=78.200(8)^{\circ}$, $\mathrm{V}=1304.8(3) \AA^{3} ; \mathrm{T}=23.0^{\circ} \mathrm{C} ; \mathrm{Z}=4 ; \mu(\mathrm{MoK} \alpha)=4.01 \mathrm{~cm}^{-1} ; 7974$ reflections measured, 5080 unique $\left(R_{\mathrm{int}}=0.038\right)$; final $R$ value 0.051 .

\section{1,6-Dithianthren-1-ylhexane-1,6-diol (13)}

Anhyd THF (15 mL) and 1,4-dibromobutane (48.8 mg, $0.41 \mathrm{mmol})$ were added sequentially to $\mathrm{Mg}$ turnings $(29.8 \mathrm{mg}, 1.23 \mathrm{mmol})$ in a dry flask, and the suspension was stirred for $1 \mathrm{~h}$ until no more $\mathrm{Mg}$ turnings dissolved. A solution of aldehyde $2 \mathrm{a}(100 \mathrm{mg}, 0.41 \mathrm{mmol})$ in THF $(10 \mathrm{~mL})$ was added to the suspension, and the mixture was stirred for $1 \mathrm{~h}$. The reaction was then quenched with $\mathrm{H}_{2} \mathrm{O}(15 \mathrm{~mL})$, and $\mathrm{CHCl}_{3}(35 \mathrm{~mL})$ was added to the mixture. The organic layer was separated, washed successively with $\mathrm{H}_{2} \mathrm{O}(2 \times 20 \mathrm{~mL})$ and brine $(2 \times 20 \mathrm{~mL})$, dried $\left(\mathrm{MgSO}_{4}\right)$, and concentrated under vacuum to give a solid product. The product was purified by TLC [silica gel, EtOAc-hexane (4:2) to give a colorless solid; yield: $70.4 \mathrm{mg}(63 \%)$; $\mathrm{mp} 147^{\circ} \mathrm{C}$ (EtOAc-hexane).

IR (KBr): 3402 (br), 2931, 1446, $1410 \mathrm{~cm}^{-1}$.

${ }^{1} \mathrm{H}$ NMR $\left(400 \mathrm{MHz}, \mathrm{CDCl}_{3}\right): \delta=1.50-1.65(\mathrm{~m}, 4 \mathrm{H}), 1.78-1.83(\mathrm{~m}$, $4 \mathrm{H}), 5.33(\mathrm{t}, J=6.0 \mathrm{~Hz}, 2 \mathrm{H}), 7.19-7.27(\mathrm{~m}, 6 \mathrm{H}), 7.42-7.52(\mathrm{~m}, 8$ $\mathrm{H})$.

${ }^{13} \mathrm{C} \mathrm{NMR}\left(100 \mathrm{MHz}, \mathrm{CDCl}_{3}\right): \delta=25.5,25.7,38.1,38.2,71.3,71.4$, 124.9, 125.0, 127.5, 127.7, 127.9, 128.0, 128.7, 129.1, 133.5, 135.1, 136.0, 136.7, 144.6

HRMS (EI): $m / z$ calcd for $\mathrm{C}_{30} \mathrm{H}_{26} \mathrm{O}_{2} \mathrm{~S}_{4}: 546.0816$; found: 546.0789 .

\section{$5,6,7,8,9,10$-Hexahydro-1,14-epithiodibenzo $[b, j]$ thiacyclounde-} cine-5,10-diol (14)

Anhyd THF $(18.4 \mathrm{~mL})$ and 1,4-dibromobutane $(22 \mu \mathrm{L} \mathrm{mg}, 0.18$ mmol) were added sequentially to $\mathrm{Mg}$ turnings (13.9 mg, 0.55 $\mathrm{mmol}$ ) in a dry flask, and the suspension was stirred for $1.5 \mathrm{~h}$ until no more of the $\mathrm{Mg}$ turnings dissolved. A solution of dialdehyde $\mathbf{1 1}$ $(50 \mathrm{mg}, 0.18 \mathrm{mmol})$ in THF $(5 \mathrm{~mL})$ was added, and the mixture was stirred for $10 \mathrm{~h}$. The reaction was quenched with $\mathrm{H}_{2} \mathrm{O}(8 \mathrm{~mL})$, and the mixture was stirred for $1 \mathrm{~h}$. The reaction was again quenched with $\mathrm{H}_{2} \mathrm{O}(8 \mathrm{~mL})$, and $\mathrm{CHCl}_{3}(25 \mathrm{~mL})$ was added to the mixture. The organic layer was separated, washed successively with $\mathrm{H}_{2} \mathrm{O}(2 \times 20$ $\mathrm{mL})$ and brine $(2 \times 20 \mathrm{~mL})$, dried $\left(\mathrm{MgSO}_{4}\right)$, and concentrated under vacuum to give a solid product. The product was purified by TLC [silica gel, $\mathrm{CH}_{2} \mathrm{Cl}_{2}$-hexane (5:1)] to give a colorless solid; yield: $11.5 \mathrm{mg}$ (19\%); $\mathrm{mp} 231-234{ }^{\circ} \mathrm{C}\left(\mathrm{CH}_{2} \mathrm{Cl}_{2}\right.$-hexane).

IR (KBr): 3319 (br), 2937, 1402, $1014 \mathrm{~cm}^{-1}$.

${ }^{1} \mathrm{H}$ NMR (400 MHz, acetone- $d_{6}$ ): $\delta=1.27-1.33$ (m, $\left.2 \mathrm{H}\right), 1.49-1.57$ (m, $2 \mathrm{H}), 1.65-1.70(\mathrm{~m}, 2 \mathrm{H}), 1.92-2.01(\mathrm{~m}, 2 \mathrm{H}), 5.60\left(\mathrm{dd}, J_{1}=2.4\right.$ $\left.\mathrm{Hz}, J_{2}=10 \mathrm{~Hz}, 2 \mathrm{H}\right), 7.35(\mathrm{t}, J=7.8 \mathrm{~Hz}, 2 \mathrm{H}), 7.52\left(\mathrm{dd}, J_{1}=1.6 \mathrm{~Hz}\right.$, $\left.J_{2}=7.8 \mathrm{~Hz}, 2 \mathrm{H}\right), 7.64\left(\mathrm{dd}, J_{1}=1.6 \mathrm{~Hz}, J_{2}=7.8 \mathrm{~Hz}, 2 \mathrm{H}\right)$. 
${ }^{13} \mathrm{C}$ NMR $\left(100 \mathrm{MHz}\right.$, acetone- $\left.d_{6}\right): \delta=25.7,40.6,72.3,124.9,128.3$, $128.4,133.6,139.2,147.8$.

HRMS (EI): $m / z$ calcd for $\mathrm{C}_{18} \mathrm{H}_{18} \mathrm{O}_{2} \mathrm{~S}_{2}: 330.0748$; found 330.0750 .

\section{Acknowledgment}

The authors express their gratitude to Toyama University and Scientific Research Grant No. 052207 from the Ministry of Education, Science, Sports and Culture of Japan for providing funding.

\section{References}

(1) (a) Lynton, H.; Cox, E. G. J. Chem. Soc. 1956, 4886. (b) Row, I.; Post, B. Acta Crystallogr. 1956, 9, 827. (c) Row, I.; Post, B. Acta Crystallogr. 1958, 11, 372. (d) Hosoya, S. Chem. Ind. (London) 1958, 980.

(2) Howell, P. A.; Curtis, R. M.; Lipxcomb, W. N. Acta Crystallogr. 1954, 7, 498.

(3) (a) Hosoya, S.; Wood, R. G. Chem. Ind. (London) 1957, 1042. (b) Hosoya, S. Chem. Ind. (London) 1958, 159.

(c) Hosoya, S. Acta Crystallogr. 1963, 16, 310.

(4) (a) Stoss, P.; Satzinger, G. Tetrahedron Lett. 1974, 1973.

(b) Tamura, Y.; Sumoto, K.; Taniguchi, H.; Ikeda, M.

J. Org. Chem. 1973, 38, 4324. (c) Tamura, Y.; Matsushima, H.; Minamikawa, J.; Ikeda, M.; Sumoto, K. Tetrahedron
1975, 31, 3035. (d) Mani, S. R.; Shine, H. J. J. Org. Chem. 1975, 40, 2756.

(5) (a) Bandlish, B. K.; Padilla, A. G.; Shine, H. J. J. Org. Chem. 1975, 40, 2590. (b) Shine, H. J.; Kim, K. Tetrahedron Lett. 1974, 99. (c) Shine, H. J.; Silber, J. J. J. Am. Chem. Soc. 1972, 94, 1026.

(6) Gilman, H.; Swayanpati, D. R. J. Org. Chem. 1958, 23, 313.

(7) Shine, H. J.; Dais, C. F. J. Org. Chem. 1965, 30, 2145.

(8) (a) Morita, H.; Kawaguchi, H. Chem. Eur. J. 2000, 6, 3976. (b) Suwabe, S.; Okuhara, A.; Sugahara, T.; Suzuki, K.; Kunimasa, K.; Nakajima, T.; Kumafuji, Y.; Osawa, Y.; Yoshimura, T.; Morita, H. Tetrahedron Lett. 2009, 50, 1381.

(9) Ogawa, S.; Muraoka, H.; Sato, R. Tetrahedron Lett. 2006, 47,2479

(10) Lee, H. J.; Kim, K. Bull. Korean Chem. Soc. 1990, 11, 80.

(11) Lovell, J. M.; Beddoes, R. L.; Joule, J. A. Tetrahedron 1996, 52,4745 .

(12) Lovell, J. M.; Joule, J. A. J. Chem. Soc., Perkin Trans. 1 1996, 2391.

(13) Crystallographic data for compounds 6, 7, and 12 have been deposited with the accession numbers CCDC 681486, 681485 , and 681484 , respectively, and can be obtained free of charge from the Cambridge Crystallographic Data Centre, 12 Union Road, Cambridge CB2 1EZ, UK, Fax: +44(1223)336033, E-mail: deposit@ccdc.cam.ac.uk, Web site: www.ccdc.cam.ac.uk/conts/retrieving.html. 